\title{
SEgmentation of Magnetic REsonance Brain TUMOR USING INTEGRATED FUZZY K-MEANS Clustering
}

\author{
P.Pedda Sadhu Naik ${ }^{1}$ and T.Venu Gopal ${ }^{2}$ \\ ${ }^{1}$ Research Scholar in CSE Department, JNTUK, Kakinada, India \\ ${ }^{2}$ Associate Professor, CSE Department, JNT UCE, Sulthanpur, India
}

\begin{abstract}
Segmentation is a process of partitioning the image into several objects. It plays a vital role in many fields such as satellite, remote sensing, object identification, face tracking and most importantly in medical field. In radiology, magnetic resonance imaging (MRI) is used to investigate the human body processes and functions of organisms. In hospitals, this technique has been using widely for medical diagnosis, to find the disease stage and follow-up without exposure to ionizing radiation.Here in this paper, we proposed a novel $M R$ brain image segmentation method for detecting the tumor and finding the tumor area with improved performance over conventional segmentation techniques such as fuzzy c means (FCM), K-means and even that of manual segmentation in terms of precision time and accuracy. Simulation performance shows that the proposed scheme has performed superior to the existing segmentation methods.
\end{abstract}

\section{KEYWORDS}

MR image, Tumor, Thresholding, FCM, K-means and binarization.

\section{INTRODUCTION}

In radiology, magnetic resonance imaging (MRI) [1] is used to investigate the human body processes and functions of organisms. These images can be formed by using the magnetic fields and radio waves. In hospitals, this technique has been using widely for medical diagnosis, to find the disease stage and follow-up without exposure to ionizing radiation.MRI has a broad range of applications in medical diagnosis and in all over world there are over 25,000 scanners to be in use. It has an impact on diagnosis and treatment in many specialties although the effect on improved health outcomes is uncertain. MRT is more preferable over computed tomography (CT) since it does not use any ionizing radiation, when either modality could yield the same information. The sustained increase in demand for MRI within the healthcare industry has led to concerns about effectiveness of cost and over diagnosis. Segmenting an image is an effort to group similar colors or elements of an image into a cluster or group. This can be achieved by clustering, which clusters the number of colors or elements into several clusters based on the similarity of color intensities and gray intensities of an image. Main objective of clustering an image is dominant colors extraction from the images. By extracting the information from images such as texture, color, shape and structure, the image segmentation can be very important to simplify. Because of the information extraction in any images, the segmentation has been used in many fields such as Enhancing the image, compression, retrieval systems i.e., search engines, object detection, and medical image processing [2]. 
From the past decades, there are so many approaches developed for the image segmentation. Among those, Fuzzy c-means (FCM) is a well known method and very popular clustering scheme, which will segment the image into several parts based on the membership function [4] and [5]. After FCM, the K-means algorithm has been proposed to reduce the computational complexity of FCM. Because of its ability to cluster huge data points very quickly, K-means has been widely used in many applications [4], [7], [8] and [9]. Later years the Hierarchical clustering is also widely applied for image segmentation [12], [13] and [14]. Then after, Gaussian Mixture Model has been used with its variant Expectation Maximization for segmenting the images [17] and [18].

Here in this paper, we proposed characterization of MR brain tumorusing shaft algorithm for detecting the tumor and finding the tumor areausing number of white pixels in a segmented MR image with an improved performance over conventional segmentation techniques such as fuzzy c means (FCM), K-means and even that of manual segmentation in terms of precision time and accuracy.

\section{RELATED WORK}

Manisha et. al. in [3] proposed an improved watershed segmentation algorithm, which provides better results than the manually segmented algorithms but it includes few drawbacks like oversegmentation and sensitivity to false edges. Recent years, fazel in [4] proposed a fuzzy expert approach for segmenting the tumor cells from the MR brain images. However, that the fuzzy approach has produced good segmented results but it suffers from determining the membership function, which is used to cluster the similar pixels in MR image. Fuzzy will be worked with allocation of membership function to the pixels based on the initial centroids selected from the pixels of input image, which is to be segmented. Specifically, determining the number of the cluster is a considerable limitation with FCM. Since the regions are spatially discontinuous grey level similarity is only verified. Considering the experimental study FCM is converging to local minima of the squared error criterion [4]. Then after, Mohammed et. al. in [5] proposed an efficient brain tumor detection scheme based on the combination of spatial information with fuzzy c-means which overcomes the drawback found in [4], but it takes much time to segment the tumor and will suffer from false edges. To overcome, the drawbacks of manually segmented, watershed and FCM [3], [4], and [5] clustering algorithms, Mary Praveena in [6] proposed a fusion based image segmentation using k-means clustering, which is an extension to the above mentioned algorithms and will provide the best results within less computational time. Later years, there are so many algorithms such as histogram based approach, anisotropic diffusion and FCM have been merged with the k-means and given the best performance over conventional techniques [7], [8], [9] and [10]. However, this k-Means is limited to produce only hyper spherical clusters. It depends on initial centroids. To update the new centroids the mean of the pixel values of the respective clusters need to be estimated. The floating values obtained in some iteration are not favorable. Significantly, positive integers or scalars are required to replace the new centroids. Hence with K-means algorithm the optimal solution is difficult to achieve. In order to overcome the drawbacks of k-means, Barakbahet. al. in [11] proposed a pillar algorithm to solve the initial centroid designation problem, by considering the pixel maximization i.e., select maximum pixel value for centroid. Authors in [19] and [20] also proposed hybrid algorithms for detecting the tumor, but they were failed to detect the tumor with higher accuracy.However, the above mentioned algorithms have the drawbacks like more computational time, less accuracy and inaccurate area estimation.

\section{Proposed Algorithm}

Here in the proposed clustering algorithm, we optimized the k-means clustering by applying fuzzy algorithm. 


\subsection{K-means clustering}

1. First we will select the number of centroids randomly i.e., depends on number of clusters

2. Now, partition the objects within each cluster

3. It finds partitions such that pixels within each cluster are as close to each other as possible, and as far from objects in other clusters as possible.

4. The objects are in the cluster or not will be calculated by measuring the distance between the cluster pixels. When the calculated Euclidean distance has minima value then the pixels will be grouped with the respective cluster.

5. Do the above process for remaining clusters also. Then, we will get three clusters with their similar pixels.

6. Now, calculate the mean of each cluster and replace the mean values with the centroids

7. Repeat the same process with these new centroids by giving the number of iterations until unless the convergence occurrence i.e., the mean value of clusters $=$ cluster centroid value.

Flow chart of K-means clustering algorithm below Figure 1.

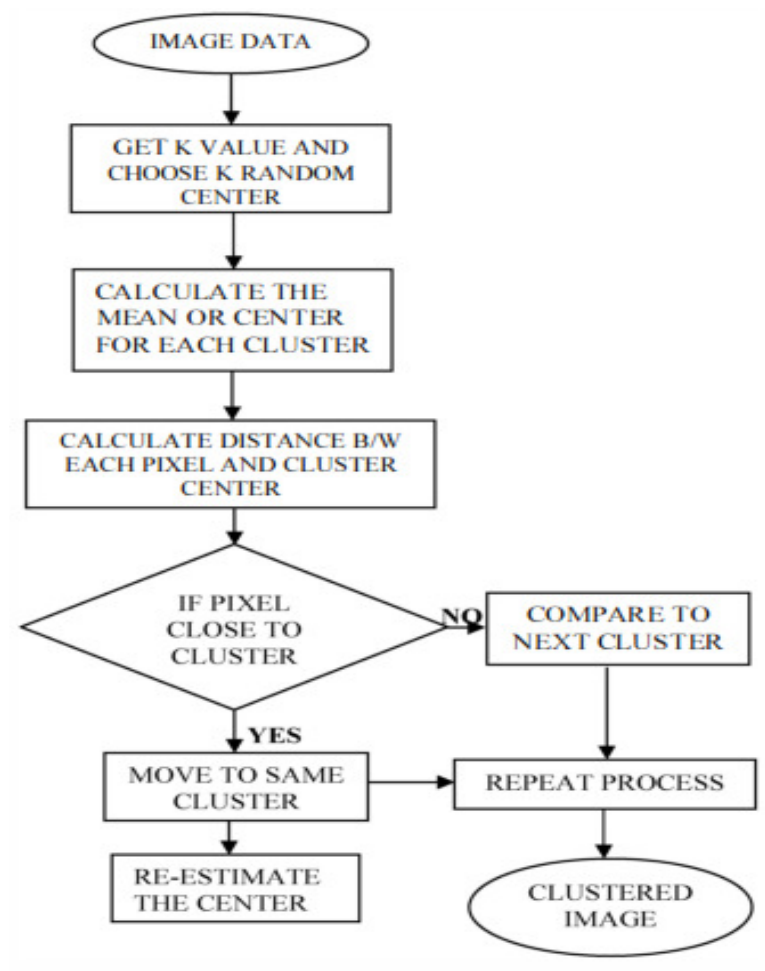

Figure 1. Flow chart of K-means clustering

\subsection{Hybrid FKM Algorithm}

Here in this section, we described our proposed hybrid fuzzy k-means (FKM) clustering in brief. First, the preprocessing has been done using median filter, which is used to remove the noise from digital images and will improve the quality of the image. Then the output of first stage will be given to the k-means clustering which gives the segmented output of de-noised image. Now, fuzzy clustering will be applied for the k-means segmented output to improve the segmentation accuracy and exact detection of tumor from MR brain images. Finally, binarization will be used to calculate the size of the tumor based on typography and digital imaging units [21]. 


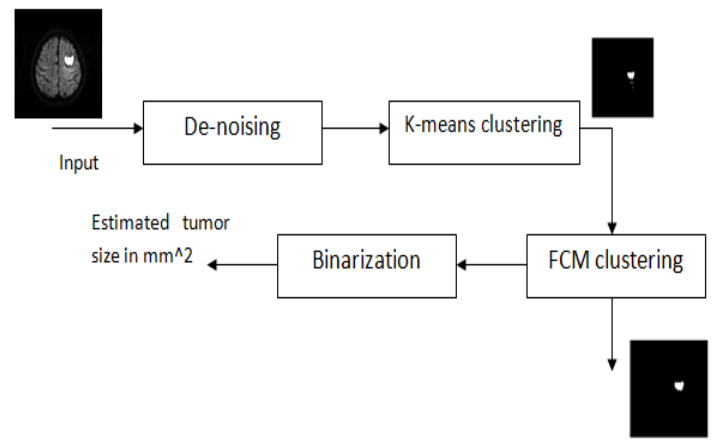

Figure 2. Proposed hybrid clustering algorithm

As mentioned in 3.1 section, while calculating the mean of cluster centroid pixels sometimes we might get the floating values, but the pixel values in an image will always be integers which does not have decimal values. Hence, we proposed a novel algorithm in figure 2 to fix this error. In the proposed approach, segmented k-means output will be further segmented by fuzzy clustering for improved accuracy. Then after, the binarization method will be applied to calculate the size of tumor which has been detected by using proposed hybrid clustering algorithm.

\subsection{Binarization}

Binarization is used to calculate the tumor area. Here, we considered the images of size 256 x 256 and the pixels in the segmented image having only two values i.e., either black or white, where the pixel value 0 denotes the black and 1 denotes the white. Hence, we can represent the segmented output image as a summation of total number of white and black pixels.

$$
\begin{gathered}
M=\sum_{x=1}^{L} \sum_{y=1}^{L}\left[f_{x, y}(0)+f_{x, y}(1)\right], \text { where } \mathrm{L}=1,2,3 \ldots 256 \\
f_{x, y}(0)=\text { black pixel having the value of zero, } \\
f_{x, y}(1)=\text { white pixels having the value of one } \\
P=\sum_{i=1}^{L} \sum_{j=1}^{L} f_{x, y}(1)
\end{gathered}
$$

Where,

$$
\mathrm{P}=\text { number of white pixels }
$$

Now, by using the above equation, we can calculate the area of the segmented tumor based on the typography and digital imaging units [21], where one pixel is equal to 0.264583 millimeters. i.e., 1 pixel $=0.264583 \mathrm{~mm}$

Then the area of tumor can be expressed as follows:

$$
A_{\text {Tumor }}=(\sqrt{P}) * 0.2646 \mathrm{~mm}^{2}
$$

\section{EXPERIMENTAL RESULTS}

In this section, we had given an overview of conventional and proposed segmented results with the area of tumor. All the experiments have been done in MATLAB 2014a 32-bit version with 
4GB RAM. We tested five set of images with various sizes such as 400x400, 512x512 and $600 \times 600$, which have the different stages of tumors. Then we evaluated the performance of conventional schemes Fuzzy c means, K-means and manually segmented algorithms with the proposed shaft algorithm for characterization of MR Brain tumors. The experimental results of MRI tumor detection using proposed algorithm and existing algorithms will be shown in below figure. By comparing the results our proposed approach for brain tumor detection will be more effective, accurate and reduced computational time. Figure 3 shows that the segmented outputs of manually segmented, FCM, K-means and proposed algorithms, we can observe that the proposed algorithm has detected the tumor more effectively with less computational time as shown in table-1.

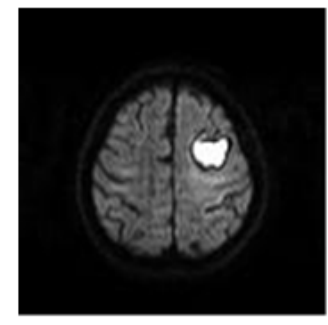

(a)

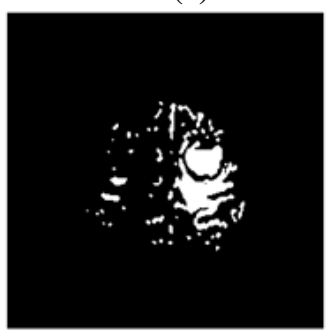

(c)

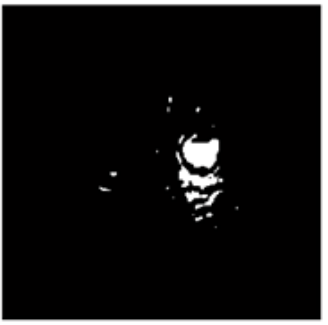

(b)

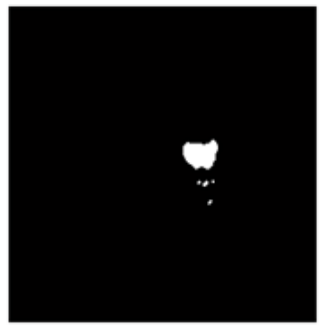

(d)

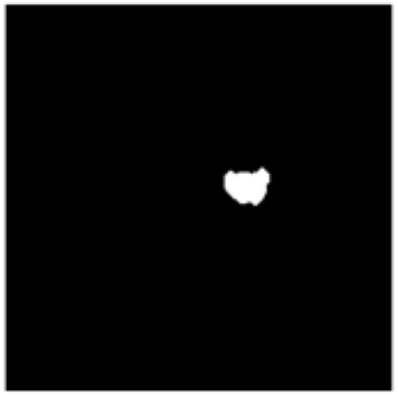

(e)

Figure 3. (a) Original Image (b) Manually Segmented Image (c) Fuzzy C Means Algorithm(d) K-means segmented image (e) Proposed method

In figure 4, we can see that the CPU computational time of existing and proposed algorithms for sample 1. It shows that the proposed algorithm has taken 0.2496 seconds to detect the tumor accurately as shown in fig 2 (e), and the existing algorithms have taken 11.2633 and 0.4056 seconds respectively. Area of tumor had given in figure 5, it shows that the proposed algorithm has got the accurate area compared with the existing algorithms FCM, k-means and even manual segmentation. 
International Journal of Computer Science \& Information Technology (IJCSIT) Vol 8, No 1, February 2016

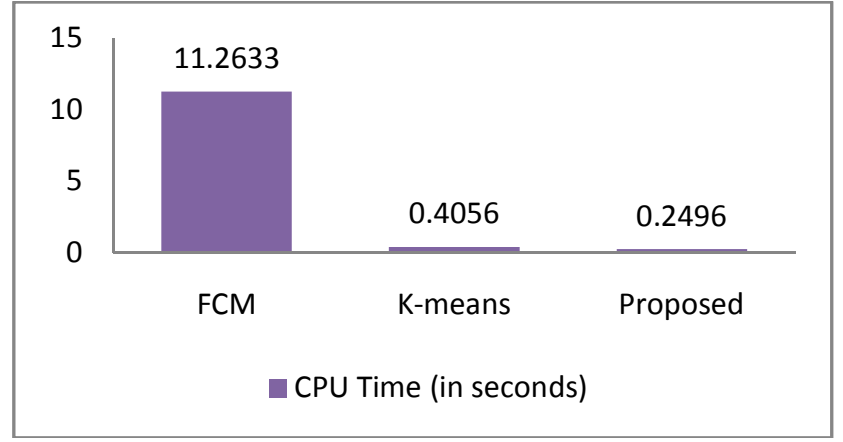

Figure 4 CPU time comparison of existing and proposed algorithms for sample 1

Table-1 Existing and proposed algorithms computational time (in seconds)

\begin{tabular}{|c|c|c|c|c|c|c|}
\hline \multirow{2}{*}{$\begin{array}{l}\text { S. } \\
\text { No. }\end{array}$} & \multirow{2}{*}{$\begin{array}{l}\text { Cluster } \\
\text { algorithm }\end{array}$} & \multicolumn{3}{|c|}{ CPU Computation time (Seconds) } & & \\
\hline & & Sample1 & Sample2 & Sample3 & Sample4 & Sample5 \\
\hline 1 & Fuzzy C Means & 11.2633 & 3.4008 & 2.9328 & 2.9172 & 4.4772 \\
\hline 2 & K-Means & 0.4056 & 0.5148 & 0.4368 & 0.3432 & 0.9828 \\
\hline 3 & Proposed & 0.2496 & 0.2340 & 0.2652 & 0.2808 & 0.2496 \\
\hline
\end{tabular}

Table-2 Proposed and Conventional algorithms Tumor area in $\mathrm{mm}^{2}$

\begin{tabular}{|c|c|c|c|c|c|c|}
\hline \multirow{2}{*}{$\begin{array}{c}\text { S. } \\
\text { No. }\end{array}$} & \multirow[b]{2}{*}{ Clustered algorithm } & \multicolumn{3}{|c|}{ Area of the tumor $\left(\mathrm{mm}^{\wedge} 2\right)$} & \multirow[b]{2}{*}{ Sample4 } & \multirow[b]{2}{*}{ Sample5 } \\
\hline & & Sample1 & Sample2 & Sample3 & & \\
\hline 1 & Manual Segmentation & 8.8035 & 18.3038 & 18.8829 & 19.6782 & 22.6656 \\
\hline 2 & Fuzzy C-Means & 13.6746 & 13.5954 & 13.4899 & 13.1497 & 18.9235 \\
\hline 3 & K-Means & 6.1291 & 13.7077 & 13.5363 & 13.2053 & 19.0721 \\
\hline 4 & Proposed method & 6.1462 & 12.1898 & 12.3602 & 11.6758 & 14.0442 \\
\hline
\end{tabular}

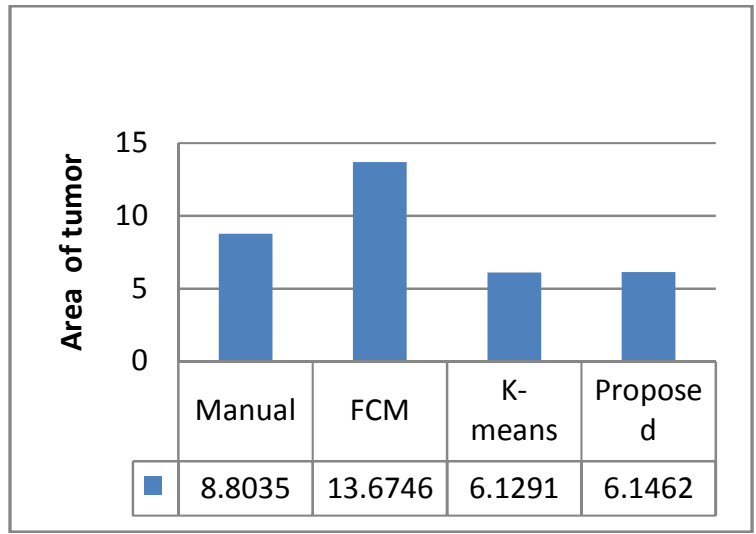

Figure 5 Comparison of tumor area for sample 1 


\section{CONCLUSION}

Here in this paper, we had proposed a novel MR brain image segmentation for detecting the tumor and to find the area of the tumor with improved accuracy and reduced computational time. This paper deals with the new shaft algorithm for reducing the computational time and binarization method to calculate the area interms of $\mathrm{mm}^{2}$ based on the typography and digital imaging units. We compared the simulation results with the existing algorithms with the proposed shaft algorithm then after we found the area of tumor and calculated the CPU computational time. Finally, the proposed algorithm has performed far better than the existing algorithms with reduced computational time.

\section{REFERENCES}

1. https://en.wikipedia.org/wiki/Magnetic_resonance_imaging

2. https://en.wikipedia.org/wiki/Image_segmentation

3. Manisha Bhagwatl, R.K.Krishna\&V.E.Pise, "Image Segmentation by Improved Watershed Transformation in Programming Environment MATLAB" International Journal of Computer Science \& Communication Vol. 1, No. 2, pp. 171-174, 2010.

4. M.H. Fazel Zarandia, M. Zarinbala, M. Izadi, "Systematic image processing for diagnosing brain tumors: A Type-II fuzzy expert system approach," Applied soft computing, pp: 285-294, 2011

5. S. ZulaikhaBeeviM, Mohamed Sathik, "An Effective Approach for Segmentation of MRI Images: Combining Spatial Information with Fuzzy C-Means Clustering" European Journal of Scientific Research, Vol. 41, No.3, pp.437-451, 2010.

6. S. Mary Praveena, Dr.I1aVennila, "Optimization Fusion Approach for Image Segmentation Using KMeans Algorithm" International Journal of Computer Applications, Vol 2, No.7, June 2010.

7. M. Masroor Ahmed \&Dzulkifli Bin Mohammad, "Segmentation of Brain MR Images for Tumor Extraction by Combining K-means Clustering and Perona-Malik Anisotropic Diffusion Model" International Journal of Image Processing, Vol. 2, No. 1, 2010

8. Tse-Wei Chen, Yi-Ling Chen, Shao-Yi Chien, "Fast Image Segmentation Based on K-Means Clustering with Histograms in HSV Color Space" Journal of Scientific Research, Vol. 44 No.2, pp.337-351, 2010.

9. Anil Z Chitade, " Colour based image segmentation using k-means clustering" International Journal of Engineering Science and Technology Vol. 2(10), 5319-5325, 2010.

10. Selvakumar, J., Lakshmi, A., Arivoli, T., "Brain Tumor segmentation and its area Calculation in Brain MR images using K-means Clustering and Fuzzy C-means algorithm", International Conference on Advances in Engineering, Science and Management (ICAESM), pp: 186-190, 2012.

11. Barakbah, A.R., Kiyoki. Y., “A Pillar algorithm for K-means Optimization by Distance Maximization for Initial Centroid Designation”, IEEE Symposium on Computational Intelligence and Data Mining, pp: 61-68, 2009.

12. A.M. Usó, F. Pla, P.G. Sevila, "Unsupervised Image Segmentation Using a Hierarchical Clustering Selection Process", Structural, Syntactic, and Statistical Pattern Recognition, Vol. 4109, pp. 799-807, 2006.

13. A.Z. Arifin, A. Asano, "Image segmentation by histogram thresholding using hierarchical cluster analysis", Pattern Recognition Letters, Vol. 27, no. 13, pp. 1515-1521, 2006.

14. B. Micušík, A. Hanbury, “Automatic Image Segmentation by Positioning a Seed*”, ECCV 2006, Part II, LNCS 3952, Springer Berlin/Heidelberg, pp. 468-480, 2006.

15. J. Chen, J. Benesty, Y.A. Huang, S. Doclo, "New Insights Into the Noise Reduction Wiener Filter", IEEE Transactions on Audio, Speech, and Language Processing, Vol. 14, No. 4, 2006.

16. Y. Pan, J.D. Birdwell, S.M. Djouadi, "Bottom-Up Hierarchical Image Segmentation Using Region Competition and the Mumford-Shah Functional", Proc. 18th International Conference on Pattern Recognition (ICPR), Vol. 2, pp. 117-121, 2006.

17. C. Carson, H. Greenspan, "Blob world: Image Segmentation Using Expectation-Maximization and Its Application to Image Querying”, IEEE Transactions On Pattern Analysis And Machine Intelligence, Vol. 24, No. 8, pp. 1026-1038, 2002.

18. C.J. Veenman, M.J.T. Reinders, E. Backer, “A maximum variance cluster algorithm”, IEEE Transactions on Pattern Analysis and Machine Intelligence, Vol. 24, No. 9, pp. 1273-1280, 2002. 
19. Alan Jose, S. Ravi, M. Sambath, "Brain tumor Segmentation using k-means clustering and Fuzzy cmeans clustering And Its Area estimation”, International Journal of Innovation Research in Computer and Communication Engineering (IJIRCCE), Vol. 2, No. 3, 2014.

20. Eman Abdel-Maksoud, Mohammed Elmogy, Rashid Al Awadhi, "Brain Tumor Segmentation based on a Hybrid Clustering Technique”, Egyptian Informatics Journal, Vol. 16, No. 1, 2015.

21. http://www.unitconversion.org/typography/millimeters-to-pixels-X-conversion.html

\section{AUTHORS}

P.Pedda Sadhu Naik received B.Tech (CSE) Degree from JNTUniversity in 2003 and M.Tech (CS) Degree from JNTUCE Anatapur in 2007.He is doing Ph D from JNTUK, Kakinada in Computer Science under the guidance of Dr T. Venu Gopal. He has 12 years of teaching experience. He joined as Assistant Professor in Dr.Samuel George Institute of Engineering \& Technology, Markapur, India in 2003. Presently he is working as Associate Professor and Head of the Department of CSE. His Interested research areas are Image Processing and Soft Computing. He has life member of ISTE and IAENG. He organized various National level Technical

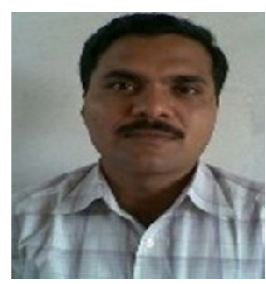
Symposiums as Convener. He attended Various National and International Workshops and Conferences on image Processing.

Dr.T.Venu Gopal received B.Tech (ECE) Degree from Osmania University Hyderabad in 1994 and M.Tech (CSE) Degree from JNTUH Hyderabad in 2003.He completed Ph D from JNTUH, Hyderabad in Computer Science. He is Associate Professor, JNTUH College of Engineering, Jagityal. arimnagar District. He has $15 y e a r s$ of teaching experience. He has worked in different colleges in different levels. Presently he is working as Associate Professor in JNTUHCE Jagityala. His Interested research areas are Image Processing and Soft Computing. He has life member of ISTE and IAENG. He published more than 30 international journals. He organized various National level Technical Symposiums as Convener. He attended

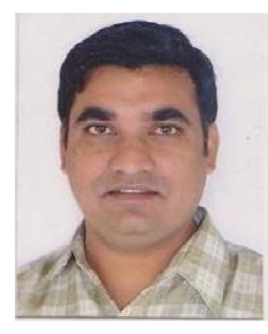
Various National and International Workshops and Conferences on image Processing. 